This is an electronic reprint of the original article. This reprint may differ from the original in pagination and typographic detail.

\author{
Author(s): Petrache, C. M.; Ragnarsson, I.; Naïdja, H.; Nowacki, F.; Leguillon, R.; Zerrouki, T.; \\ Greenlees, Paul; Jakobsson, Ulrika; Jones, Peter; Julin, Rauno; Juutinen, Sakari; \\ Ketelhut, Steffen; Leino, Matti; Nyman, Markus; Peura, Pauli; Rahkila, Panu; \\ Ruotsalainen, Panu; Sarén, Jan; Scholey, Catherine; Sorri, Juha; Uusitalo, Juha
}

Title: $\quad$ Search for the terminating 27 - state in $140 \mathrm{Nd}$

Year: $\quad 2015$

Version:

Please cite the original version:

Petrache, C. M., Ragnarsson, I., Naïdja, H., Nowacki, F., Leguillon, R., Zerrouki, T., Greenlees, P., Jakobsson, U., Jones, P., Julin, R., Juutinen, S., Ketelhut, S., Leino, M., Nyman, M., Peura, P., Rahkila, P., Ruotsalainen, P., Sarén, J., Scholey, C., . . Uusitalo, J. (2015). Search for the terminating 27- state in 140Nd. Physical Review C, 92(3), Article 034314. https://doi.org/10.1103/PhysRevC.92.034314

All material supplied via JYX is protected by copyright and other intellectual property rights, and duplication or sale of all or part of any of the repository collections is not permitted, except that material may be duplicated by you for your research use or educational purposes in electronic or print form. You must obtain permission for any other use. Electronic or print copies may not be offered, whether for sale or otherwise to anyone who is not an authorised user. 


\title{
Search for the terminating $27^{-}$state in ${ }^{140} \mathrm{Nd}$
}

C. M. Petrache, ${ }^{1}$ I. Ragnarsson, ${ }^{2}$ H. Naïdja,${ }^{3,4,5}$ F. Nowacki,${ }^{3}$ R. Leguillon, ${ }^{1,6}$ T. Zerrouki, ${ }^{7}$ P. T. Greenlees, ${ }^{7}$ U. Jakobsson,,${ }^{7,8}$ P. Jones, ${ }^{7,9}$ R. Julin, ${ }^{7}$ S. Juutinen, ${ }^{7}$ S. Ketelhut, ${ }^{7}$ M. Leino, ${ }^{7}$ M. Nyman, ${ }^{7,10}$ P. Peura, ${ }^{7}$ P. Rahkila, ${ }^{7}$ P. Ruotsalainen, ${ }^{7,11}$ J. Saren, ${ }^{7}$ C. Scholey, ${ }^{7}$ J. Sorri, ${ }^{7}$ and J. Uusitalo ${ }^{7}$

${ }^{1}$ Centre de Sciences Nucléaires et de Sciences de la Matière, Université Paris-Sud and CNRS/IN2P3, 91405 Orsay, France

${ }^{2}$ Division of Mathematical Physics, LTH, Lund University, Post Office Box 118, SE-221 00 Lund, Sweden

${ }^{3}$ Universitè de Strasbourg, IPHC, 23 rue du Loess, 67037 Strasbourg, France, and CNRS, UMR7178, 67037 Strasbourg, France

${ }^{4}$ GSI Helmholtzzentrum für Schwerionenforschung GmbH, 64291 Darmstadt, Germany

${ }^{5}$ Université Constantine 1, Route Ain-El bey, 25000 Constantine, Algérie

${ }^{6}$ Advanced Science Research Center, Japan Atomic Energy Agency, 2-4 Shirakata Shirane, Tokai, Ibaraki 319-1195, Japan

${ }^{7}$ Department of Physics, University of Jyväskylä, Jyväskylä FIN-40014, Finland

${ }^{8}$ Department of Physics, Royal Institute of Technology (KTH), Alba Nova University Center, SE-10691 Stockholm, Sweden

${ }^{9}$ Department of Nuclear Physics, iThemba Laboratory for Accelerator Based Sciences, Post Office Box 722, Somerset West 7129, South Africa

${ }^{10}$ Institute for Reference Materials and Measurements (IRMM), 2440 Geel, Belgium

${ }^{11}$ TRIUMF, 4004 Wesbrook Mall, Vancouver, British Columbia, Canada V6T 2A3

(Received 1 March 2015; revised manuscript received 13 August 2015; published 14 September 2015)

\begin{abstract}
In the search for the fully aligned $27^{-}$state in ${ }^{140} \mathrm{Nd}$ predicted by cranked Nilsson-Strutinsky calculations, new close-to-spherical high-spin states have been discovered. Both the close-to-spherical and the triaxial calculated states are in good agreement with the experimental results, supporting the existence of shape coexistence up to very high spins. Shell-model calculations using a newly developed effective interaction for the $50 \leqslant N, Z \leqslant 82$ mass region are in good agreement with the observed spherical states. The comparison between the experimental and calculated level energies allowed the relative energy to be established between several proton and neutron orbitals at high energy and spins.
\end{abstract}

DOI: 10.1103/PhysRevC.92.034314

PACS number(s): 21.10.Re, 21.60.Ev, 23.20.Lv, 27.60.+j

\section{INTRODUCTION}

The nuclei with a few holes in the $N=82$ shell closure are spherical or oblate deformed at low spins [1]. They can get more deformed or have isomeric states based on simple particle-hole excitations at high spins, and are thus of utmost importance for testing the various nuclear potentials or effective interactions. This is the case for the nuclei in the $A \sim 140$ mass region, which can acquire significant axial deformation $\left(\varepsilon_{2}=0.45\right.$ for the superdeformed band of $\left.{ }^{140} \mathrm{Nd}\right)$ [2] or stable triaxial shape $\left(\varepsilon_{2}=0.2, \gamma \approx+30^{\circ}\right.$ for ${ }^{138-141} \mathrm{Nd}$ ) [3-7] in configurations involving high- $j$ neutron particles from above $N=82$. In this mass region the potential energy surfaces show profound minima at close-to-spherical shape [6-8], deep enough to allow the observation of such states up to relatively high spins. Some of these states which have configurations with the spins of all active particles aligned along the rotation axis are favored energetically and can become yrast traps with possible isomeric character. Such a situation is encountered in the ${ }^{138-141} \mathrm{Nd}$ nuclei with a few holes in $N=82$, in which isomeric states were observed at low and high spins [4,7,9-11]. At present, the highest isomer discovered in this mass region has spin $20^{+}$and was identified in ${ }^{140} \mathrm{Nd}$, being predicted previously in cranked NilssonStrutinsky (CNS) calculations [9]. Its $T_{1 / 2}=1.23(7) \mu \mathrm{s}$ lifetime supports the $20^{+}$assignment and the interpretation as a close-to-spherical configuration that coexists with the surrounding triaxial bands [10]. Also the transitions feeding the $20^{+}$isomer from higher-lying states have been identified in a recoil-tagging experiment [11] and the level scheme developed up to very high spins [6]. The present article reports the search for the next higher-lying $27^{-}$close-to-spherical state predicted in CNS calculations [5,9]. The new experimental results are compared with CNS and shell-model calculations, revealing their impact on the understanding of the high-spin nuclear structure in this mass region.

\section{EXPERIMENTAL DETAILS AND RESULTS}

High-spin states in ${ }^{140} \mathrm{Nd}$ have been populated via the ${ }^{96} \mathrm{Zr}\left({ }^{48} \mathrm{Ca}, 4 n\right)$ reaction with a beam energy of $180 \mathrm{MeV}$. The target was a ${ }^{96} \mathrm{Zr}$ self-supporting foil of $735 \mu \mathrm{g} / \mathrm{cm}^{2}$ thickness. The ${ }^{48} \mathrm{Ca}$ beam was provided by the K- 130 cyclotron at the University of Jyväskylä, Finland. The main reaction channels led to ${ }^{139} \mathrm{Nd}$ and ${ }^{140} \mathrm{Nd}$ with cross sections of around $40 \mathrm{mb}$. Details about the experimental setup and data sorting were recently published in Refs. [6,11]. A total of $5 \times 10^{9}$ recoilgated events has been collected. We produced $\gamma-\gamma$ matrices for the clovers and for the segmented planar Ge detectors of the Gamma Recoil Electron Alpha Tagging (GREAT) spectrometer [12] at the focal plane of the Recoil Ion Transport Unit (RITU) separator [13], and a $\gamma-\gamma-\gamma$ cube for JUROGAM II at the target position [14]. The transition multipolarities of the newly observed transitions have been deduced from the anisotropy ratios $W(f, b) / W\left(90^{\circ}\right)$, which for pure stretched $E 2$ and $E 1$ known transitions measured in the same reaction are 0.61(3) and 0.28(5), respectively. The level scheme of ${ }^{140} \mathrm{Nd}$ was recently published in Ref. [6]. The states and bands discussed in the present paper are shown in Fig. 1.

The experimental information on the observed transitions around the newly identified $27^{(-)}$state is reported in Table I. 


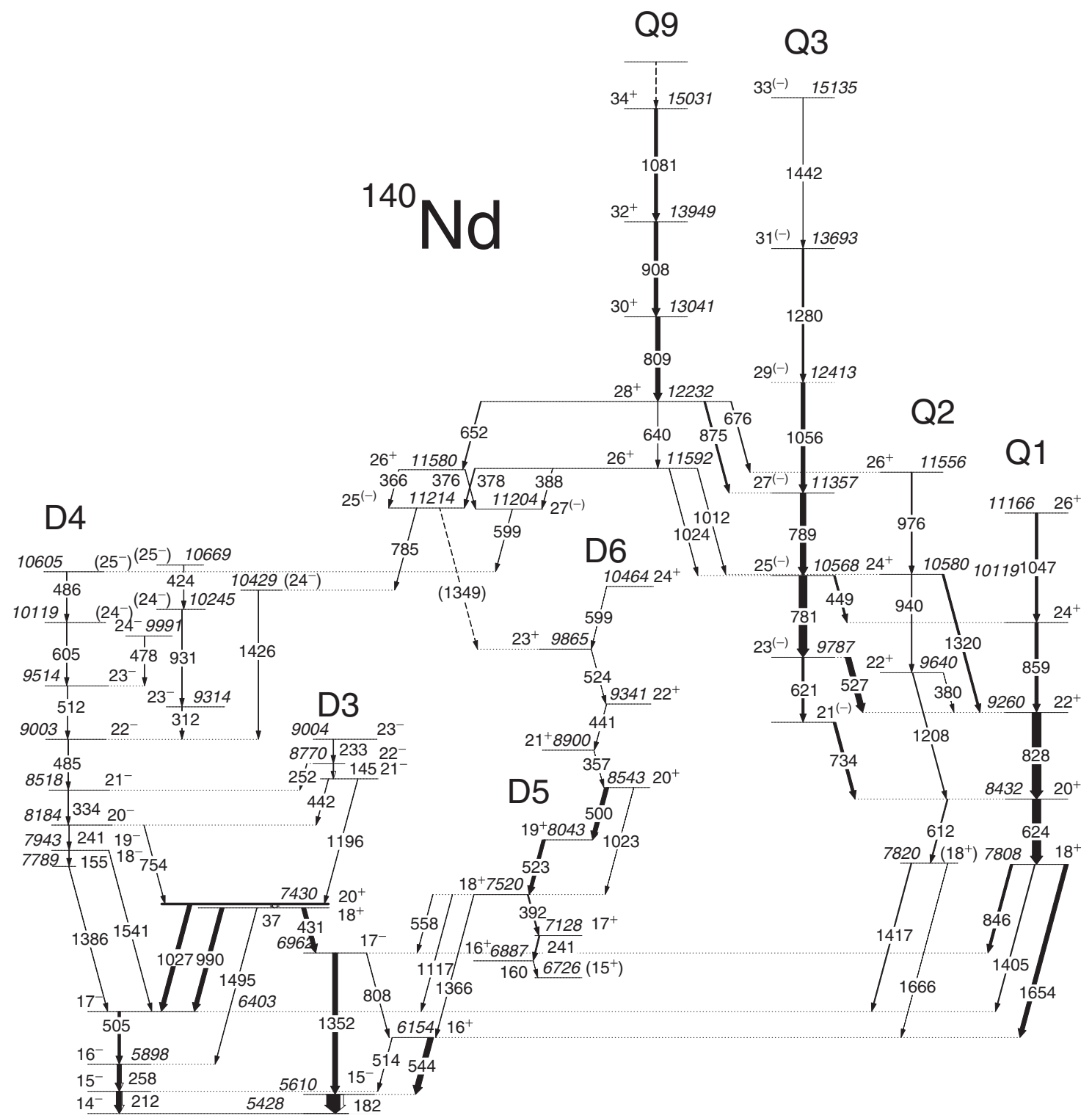

FIG. 1. Partial level scheme of ${ }^{140} \mathrm{Nd}$ showing selected states related to the decay of the $27^{(-)}$state.

The 809-, 908-, and 1081-keV transitions of band $Q 9$ are clearly observed in coincidence with carefully chosen delayed

TABLE I. Energies, anisotropies, multipolarities, and spin-parity assignments for the $\gamma$-ray transitions around the new $27^{(-)}$state in ${ }^{140} \mathrm{Nd}$.

\begin{tabular}{lccc}
\hline \hline$E_{\gamma}$ & Anisotropy & Multipolarity & $J_{i}^{\pi} \rightarrow J_{f}^{\pi}$ \\
\hline 366 & $0.26(2)$ & $E 1$ & $26^{+} \rightarrow 25^{(-)}$ \\
376 & & $(E 1)$ & $26^{+} \rightarrow 27^{(-)}$ \\
378 & $0.31(2)$ & $E 1$ & $26^{+} \rightarrow 25^{(-)}$ \\
388 & $0.28(5)$ & $E 1$ & $26^{+} \rightarrow 27^{(-)}$ \\
640 & $0.62(3)$ & $E 2$ & $28^{+} \rightarrow 26^{(+)}$ \\
652 & $0.61(3)$ & $E 2$ & $28^{+} \rightarrow 26^{(+)}$ \\
1012 & & $E 2$ & $26^{+} \rightarrow 24^{+}$ \\
1024 & & $E 1$ & $26^{+} \rightarrow 25^{(-)}$ \\
\hline \hline
\end{tabular}

transitions of ${ }^{140} \mathrm{Nd}$ detected in the GREAT spectrometer, where the residual nuclei arrive after a flight time of around 650 ns [11] (see Fig. 2). This indicates that the decay of band $Q 9$ is delayed by either the $20^{+}$isomer or by another higher-lying unidentified isomeric state. The possible existence of transitions from band $Q 9$ to the states populating the $20^{+}$isomer [11] and to the high-spin bands which can be populated by transitions de-exciting the band $Q 9$ was carefully investigated. By double-gating on the transitions of band $Q 9$, we identified a new lower-lying state at $11592 \mathrm{keV}$, which decays through the weak 1012- and 1024-keV transitions towards the $24^{+}$and $25^{(-)}$states of bands $Q 2$ and $Q 3$, respectively (see Fig. 3). The similar decay of the $26^{+}$and $28^{+}$states of band $Q 9$ towards the bands $Q 2$ and $Q 3$ suggests that the new $26^{+}$state at $11592 \mathrm{keV}$ belongs to band $Q 9$. In addition to the decay towards the known bands $Q 2$ and $Q 3$, we also identified the two parallel cascades, $652-366 \mathrm{keV}$ and 


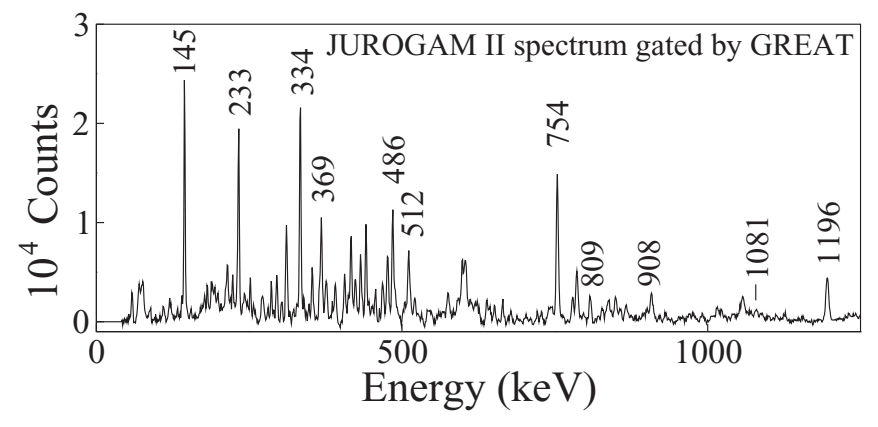

FIG. 2. Spectrum of prompt transitions measured by JUROGAM II gated with clean delayed transitions below the $20^{+}$isomer in ${ }^{140} \mathrm{Nd}$ $(188,191,258,720,868,922,1017$, and $1495 \mathrm{keV})$ measured by the clovers placed at the focal plane. The strongest transitions populate the $20^{+}$isomer [11].

640-378 keV, with relative intensities of the order of $0.5 \%$, passing through the newly identified $26^{+}$states at 11580 and $11592 \mathrm{keV}$ and leading to a $25^{(-)}$state at $11214 \mathrm{keV}$ (the spin assignment to the new states is discussed later in this section). Double-gated prompt spectra measured at the target position, with gates on the transitions of the two cascades and on the 809-keV transition of band $Q 9$ are shown in Fig. 4.

The two new $26^{+}$states at 11580 and $11592 \mathrm{keV}$ also decay towards a new $27^{(-)}$state at $11204 \mathrm{keV}$ through the 376- and $388-\mathrm{keV}$ transitions, which are roughly 4 times weaker than the $366-$ and $378-\mathrm{keV}$ transitions towards the $25^{(-)}$state at $11592 \mathrm{keV}$ (see Fig. 3).

The decay of the $25^{(-)}$and $27^{(-)}$states is very fragmented, with transition intensities below $0.1 \%$ [6], populating states above the $20^{+}$isomer, but probably also other unobserved states [11]. The analysis of the prompt $\gamma-\gamma-\gamma$ coincidences

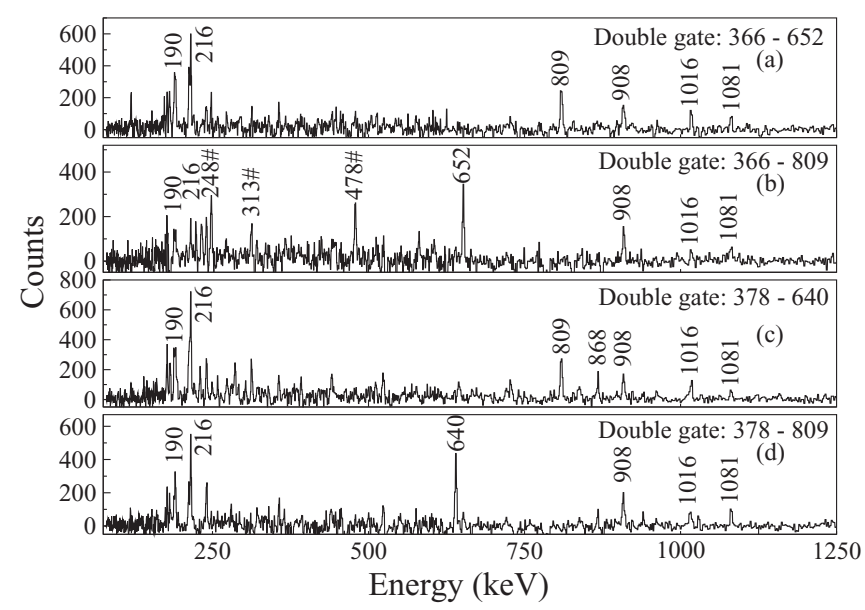

FIG. 3. Double-gated prompt coincidence spectra measured by JUROGAM II, with gates on the transitions populating the $25^{(-)}$ state and de-exciting the lowest states of band $Q 9$. Strong low-lying transitions in ${ }^{140} \mathrm{Nd}$ are indicated by their energies in $\mathrm{keV}$. The 190-keV peak corresponds to the 188- and 191-keV transitions de-exciting the $13^{-}$and $12^{-}$states, respectively, while the $216-\mathrm{keV}$ transition de-excites the $9^{-}$state [6]. The contaminating transitions are marked with \#.

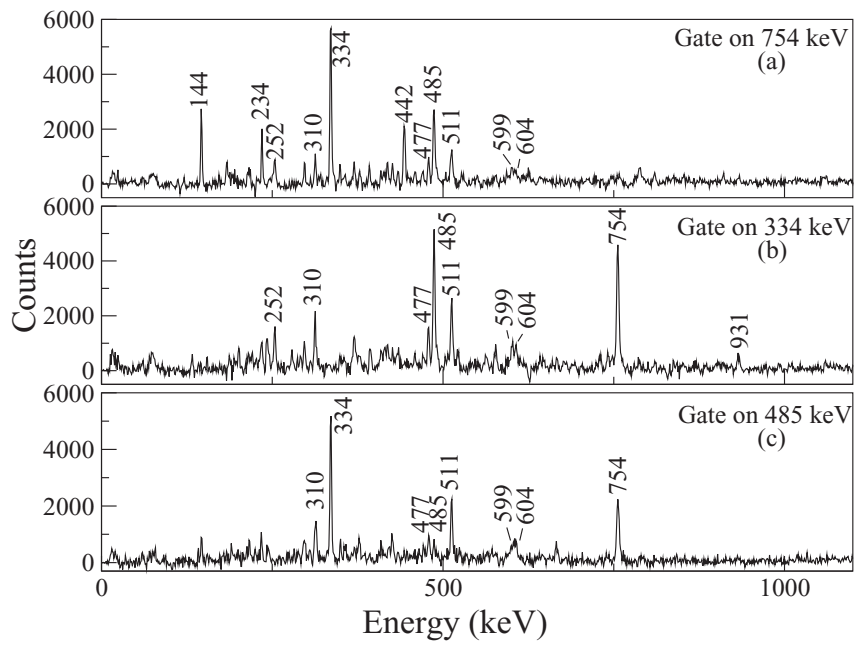

FIG. 4. Single-gated spectra from $\gamma-\gamma$ coincidences detected in JUROGAM II gated by recoils detected in the focal plane of the RITU spectrometer, showing the new transitions of 485, 604, and $931 \mathrm{keV}$ above the $20^{+}$isomer and the $599-\mathrm{keV}$ transition from the $27^{(-)}$state.

detected in JUROGAM II led to the identification of a very weak $1349-\mathrm{keV}$ tentative transition populating the $23^{+}$state of band $D 6$. By analyzing the prompt $\gamma-\gamma$ coincidences detected in JUROGAM II gated by recoils detected at the focal plane of the RITU spectrometer, we have been able to identify five new states above the $20^{+}$isomer, with energies of 10119,10245 , 10429,10605 , and $10669 \mathrm{keV}$, and the 599- and 785-keV transitions from the $27^{(-)}$and $25^{(-)}$states towards the 10605 and 10429-keV states, respectively (see Fig. 5). The fact that we could identify only three very weak transitions de-exciting the $27^{(-)}$and $25^{(-)}$states can be due to their possible isomeric character, with lifetimes significantly longer than the time resolution of the coincidence measurement, which was around $30 \mathrm{~ns}$.

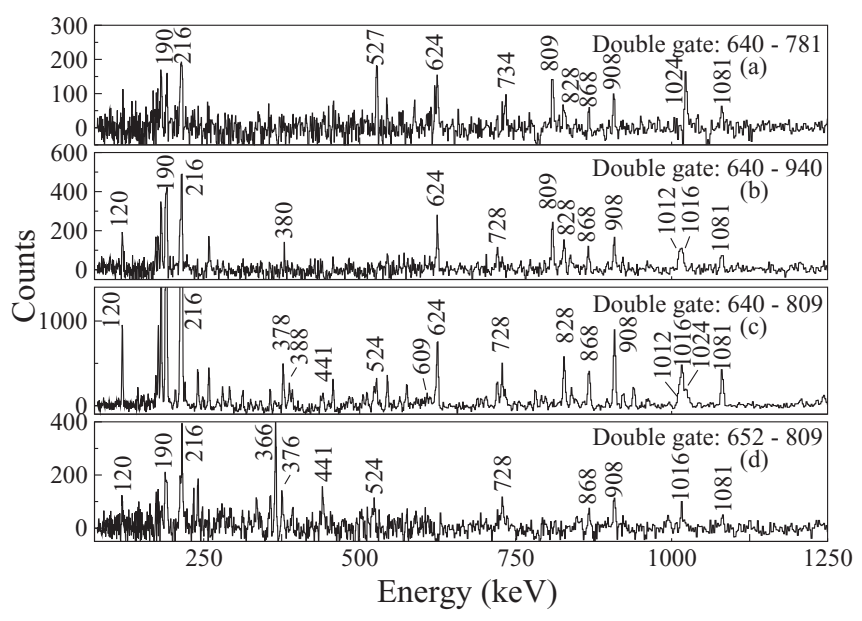

FIG. 5. Double-gated prompt coincidence spectra measured by JUROGAM II with gates on the 640- and 652-keV transitions populating the $26^{+}$states and on the 781-, 940-, and $809-\mathrm{keV}$ transitions of the bands $Q 3, Q 2$, and $Q 9$, respectively. 
A confirmation of these results was obtained from the data of a previous EUROBALL experiment using the same reaction ${ }^{96} \mathrm{Zr}\left({ }^{48} \mathrm{Ca}, 4 n\right)$ at a beam energy of $195 \mathrm{MeV}$ [6]. Spectra gated on the same transitions and additional double-gated spectra showing the same coincidence relationships as those from the JUROGAM II experiment, which support the existence of the $25^{(-)}$and $27^{(-)}$states at 11204 and $11214 \mathrm{keV}$, respectively, can be found in Ref. [15].

The $I^{\pi}=25^{(-)}$assignment for the $11214-\mathrm{keV}$ state is based on the following arguments. The spin $26^{+}$of the $11592-\mathrm{keV}$ state of band $Q 9$ is fixed by the $1012-\mathrm{keV}$ transition towards the $24^{+}$state of band $Q 2$, which must be $E 2$. The spin of the $11214-\mathrm{keV}$ state can be either 25 or 27, since the anisotropies of the $366-$ and $378-\mathrm{keV}$ transitions de-exciting the $26^{+}$states clearly indicate their $\Delta I=1$ character. However, the possible existence of the $1349-\mathrm{keV}$ transition between the $11214-\mathrm{keV}$ state and the $23^{+}$state of band $D 6$ favors the $I=25$ alternative, since it would lead to an unrealistic $E 4$ character for the $1349-\mathrm{keV}$ transition. We assign therefore spin 25 to the $11214-\mathrm{keV}$ state. The spin of the $11204-\mathrm{keV}$ state can be either 25 or 27 , since the $388-\mathrm{keV}$ transition from the $26^{+}$state at $11592 \mathrm{keV}$ has an anisotropy which clearly indicates its $\Delta I=1$ character. However, due to the much weaker population of this state we prefer the spin 27 . The spins assigned to the 11204- and 11214-keV states, together with realistic assumptions for the spins of the newly observed states above the $20^{+}$isomer, mainly based of yrastness considerations, lead to $E 2$ and $E 1$ characters for the 599and $785-\mathrm{keV}$ transitions, respectively.

The parity of the 11204- and 11214-keV states could not be established using the polarization information that can in principle be extracted from the clover detectors of the JUROGAM II array, because the populating and de-exciting transitions have very low intensity.

\section{DISCUSSION}

\section{A. CNS calculations}

The CNS model is described in Refs. [16-19]. The comparison between the CNS calculations and the experimental data are summarized in Fig. 6. The configurations can be defined relative to a ${ }^{132} \mathrm{Sn}$ core as

$$
\pi(d g)^{p_{1}}\left(h_{11 / 2}\right)^{p_{2}} v(s d)^{-n_{1}}\left(h_{11 / 2}\right)^{-n_{2}}(h f)^{n_{3}}\left(i_{13 / 2}\right)^{n_{4}},
$$

for which we use the shorthand notation $\left[p_{1} p_{2}, n_{1} n_{2}\left(n_{3} n_{4}\right)\right]$. The pseudospin partners [20] $d_{5 / 2} g_{7 / 2}(d g), s_{1 / 2} d_{3 / 2}(s d)$, and $h_{9 / 2} f_{7 / 2}(h f)$ are not distinguished in the CNS formalism. In some cases, for an odd number of particles in a group, the signature will be specified as $+(\alpha=+1 / 2)$ or $-(\alpha=-1 / 2)$. Note also that the labels do not refer to pure $j$ shells, but rather to the dominating amplitudes in the Nilsson orbitals. The $A=150$ parameters $[16,21]$ will be used for the calculations on ${ }^{140} \mathrm{Nd}$.

According to the assignments in Ref. [6], the band $Q 9$ has the configuration [82,3_2(21)]. It decays to band $Q 3$ which has a $\left[82,22\left(1_{+} 1\right)\right]$ configuration and to band $Q 2$, which is too short and irregular to be given any assignment. Band $Q 3$ decays to band $Q 1$, which has a [82,22(20)] configuration. As one can see, the configurations of bands $Q 9, Q 3$, and $Q 1$ are

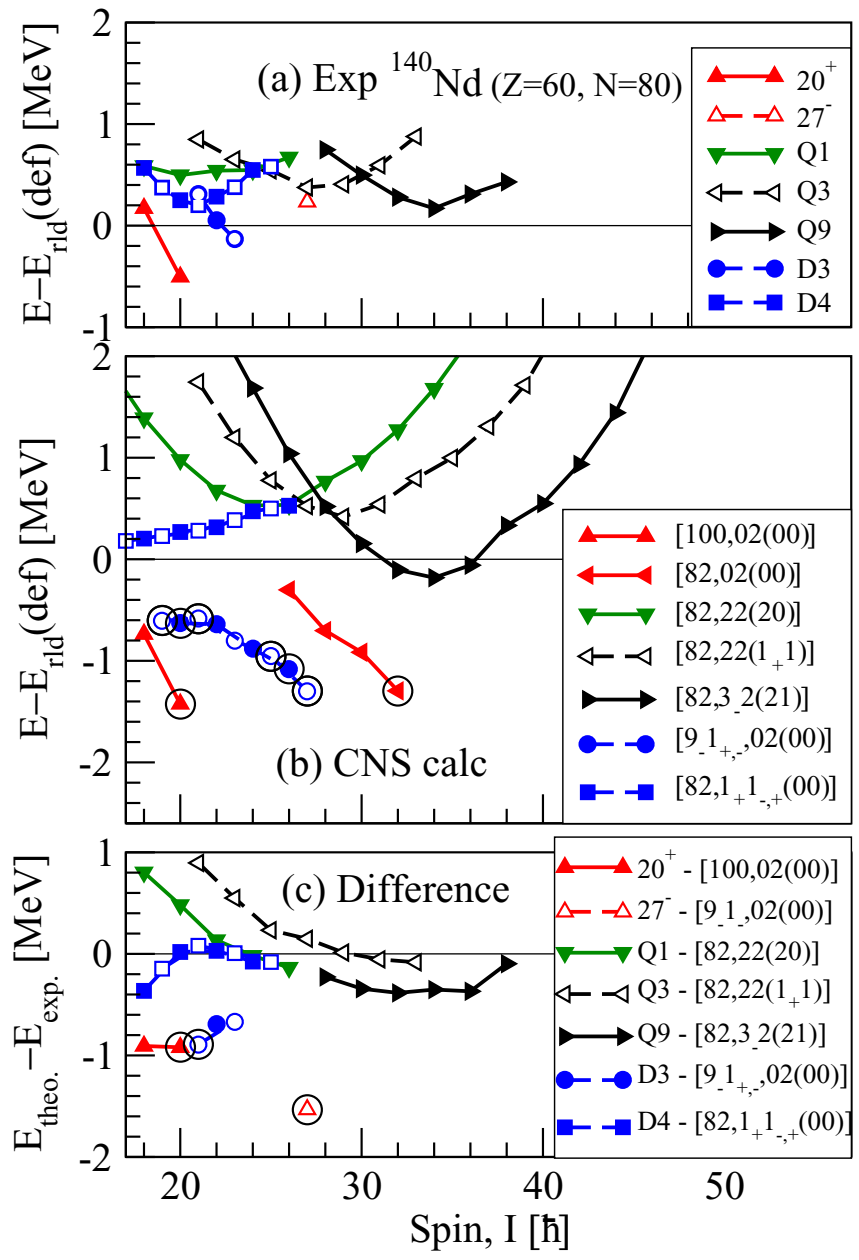

FIG. 6. (Color online) Comparison between CNS calculations and selected states of ${ }^{140} \mathrm{Nd}$ : the band $Q 9$, the bands $Q 1$ and $Q 3$ towards which $Q 9$ decays, the $20^{+}$isomer, the newly observed $27^{(-)}$ state, and the bands $D 3$ and $D 4$ which link the $27^{(-)}$and $20^{+}$states. The energy relative to a rotating liquid drop reference for the observed states is shown in the upper panel, for the calculated configurations in the middle panel, and the difference between experiment and calculations is shown in the lower panel. States of positive (negative) parity are drawn by full (dashed) lines and even (odd) spin states by closed (open) symbols. For the $D$ bands, only the closed symbols are drawn in the legends. Aligned states at $\gamma=60^{\circ}$ or $\gamma=-120^{\circ}$ are encircled. See text for more details.

related by simple particle-hole excitations, nicely accounting for the connecting transitions between these bands.

Considering also the general agreement in Fig. 6, there is strong evidence that these assignments for the $Q$ bands are correct. The present results show new decay branches of band $Q 9$ towards the $25^{(-)}$and $27^{(-)}$states at 11214 and $11204 \mathrm{keV}$, respectively, passing through intermediate $26^{(+)}$states. One possibility would then be that this $27^{(-)}$state is assigned to the $\pi\left[(d g)^{9} h_{11 / 2}^{1}\right]_{17^{-}} \otimes v\left(h_{11 / 2}^{-2}\right)_{10^{+}}\left(\left[9_{-} 1_{-}, 02(00)\right]\right.$ in CNS notation) terminating state. However, the relatively high energy of the observed $27^{(-)}$state and the large discrepancy with the CNS calculations suggest that this is not the case. 
For the $D$ bands, it turns out that the three states observed in band $D 3$ shows a down-sloping behavior when drawn relative to the rotating liquid drop energy; see Fig. 6 . This is very similar to the calculated downslope of the [91,02(00)] configuration when it approaches the terminating $27^{-}$state. Note that the two signatures are essentially degenerate in this configuration which is formed at $\gamma \approx-120^{\circ}$ (rotation around prolate symmetry axis). We suggest that band $D 3$ should be assigned to this $[91,02(00)]$ configuration, where the highest spin states $I=24-27$ are unobserved at present. Indeed, when comparing experiment and CNS calculations, the difference between the isomeric $20^{+}$state and the $[100,02(00)]$ configuration is very similar to the difference between the band D3 and the [91,02(00)] configuration (see Fig. 6), giving further evidence for the present assignments. Going to somewhat higher spin values in the CNS calculations, also the $32^{+}$state with a $\pi(d g)^{8}\left(h_{11 / 2}\right)^{2} \otimes v\left(h_{11 / 2}\right)^{-2}([82,02(00)]$ in CNS notation) configuration is yrast and very favored in energy (see Fig. 6).

The band $Q 9$ shows two decay branches to band $D 4$, so it appears relevant to consider possible assignments for band $D 4$. Because there are in total two bands of each signature for the $[91,02(00)]$ configuration, one possibility would be to assign also the band $D 4$ to this configuration. However, the upslope at the highest spin values for the $E-E_{\text {rld }}$ curve in Fig. 6 appears to exclude this possibility, because all the [91,02(00)] bands are clearly down-sloping when approaching termination at $I_{\max }=25-27$. The next possibility is then to excite another proton to $h_{11 / 2}$, i.e., to the $[82,11(00)]$ configuration, if we require negative parity with no neutrons excited across the $N=82$ gap. Indeed, as seen in Fig. 6, the difference between calculations and experiment comes out rather consistently with values close to those for the $Q$ bands, even though the downslope of the difference curve at low spin seems somewhat strange. Another possible assignments for band $D 4$ might be configurations with one neutron excited across the $N=82$ gap where the increased deformation will largely compensate for the big energy cost of the neutron excitation. Thus, the [82,12(10)] configuration, which was considered in Ref. [6], comes only around $0.5 \mathrm{MeV}$ higher in energy than the $[82,11(00)]$ configuration, which has a difference curve with similar shape. The $[82,12(10)]$ configuration has non-negligible signature, but it will probably be removed if a tilting of the spin vector is allowed [6]. Indeed, some tilting of the spin vector is expected for the configurations of the $D$ bands, which has only have a minor influence on the total energy (see Ref. [7]).

\section{B. Shell-model calculations}

Extensive shell-model (SM) calculations have been performed for the high-spin bands of ${ }^{140} \mathrm{Nd}$, using the model space $1 g_{7 / 2}, 2 d_{5 / 2}, 2 d_{3 / 2}, 3 s_{1 / 2}, 1 h_{11 / 2}$ for both protons and neutrons above the closed core ${ }^{100} \mathrm{Sn}$. The interaction GCN5082 [22] employed in the calculation of different nuclei with $50 \leqslant$ $N, Z \leqslant 82$ has been used [23,24]. Its application for the high-spin structure of ${ }^{140} \mathrm{Nd}$ constitutes a challenge for SM, which now become possible with the parrallelized versions of the NATHAN and ANTOINE codes developed recently [25].
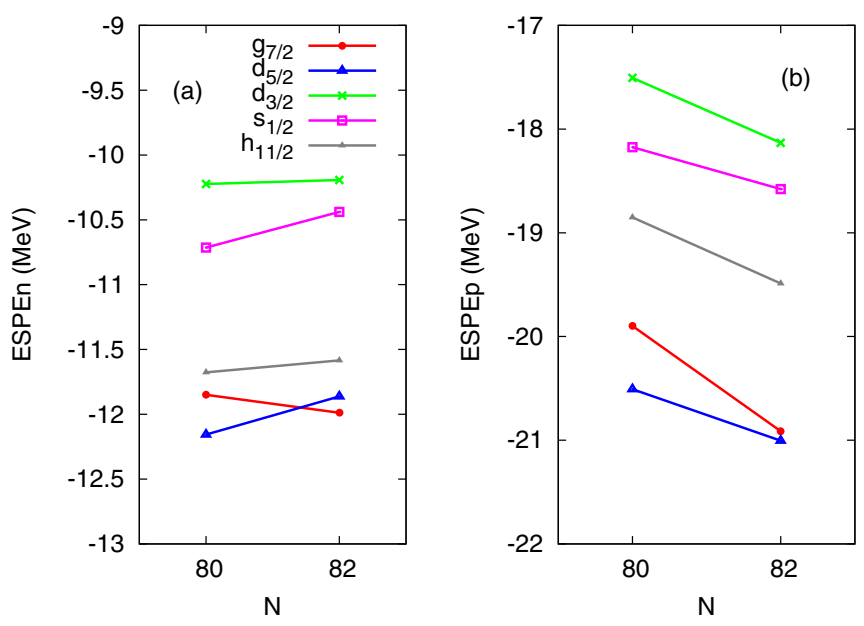

FIG. 7. (Color online) Variation of neutron (a) and proton (b) effective single-particle energies as function of the neutron number for $Z=60$.

In this work special attention has been paid to the lowestlying $20^{+}$and $27^{-}$states, corresponding to the $I_{\max }$ states of the $[100,02(00)]\left[\pi(d g)^{10} \otimes v\left(h_{11 / 2}\right)^{-2}\right]$ and $[91,02(00)]$ $\left[\pi(d g)^{9}\left(h_{11 / 2}\right)^{1} \otimes v\left(h_{11 / 2}^{-2}\right)\right]$ configurations. If, contrary to the conclusion in the CNS section above, the observed $27^{(-)}$state at $11204 \mathrm{keV}$ is assumed to correspond to the lowest $27^{-}$ state in the shell model, the calculated energies of the $20^{+}$ and $27^{-}$states are higher than the experimental values by 463 and $514 \mathrm{keV}$, respectively. However, the gap between them is reproduced with a very good precision $E_{\text {gap }}(\exp )-E_{\text {gap }}($ th $)=$ $3786-3854 \mathrm{keV}=68 \mathrm{keV}$.

In the CNS calculations, the $20^{+}$isomer has an almost pure $\pi\left(g_{7 / 2} d_{5 / 2}\right)_{10^{+}}^{10} \otimes v\left(h_{11 / 2}\right)_{10^{+}}^{2}$ configuration while the amplitude of the $\pi\left(g_{7 / 2}\right)^{6}\left(d_{5 / 2}\right)_{10^{+}}^{4} \otimes v\left(h_{11 / 2}\right)_{10^{+}}^{2}$ configuration is only $24 \%$ in the shell model. While the CNS calculations come very close to the experimental difference between the $20^{+}$and $18^{+}$states, $37 \mathrm{keV}$, this difference is much larger in the shell model, $650 \mathrm{keV}$. The SM discrepancy can be due to the energy of the $h_{11 / 2}$ orbital, which plays an important role in this nucleus. Indeed, increasing the single-particle energy of the $h_{11 / 2}$ orbital by about $200 \mathrm{keV}$ leads to an energy difference between the $20^{+}$and $18^{+}$states of about $400 \mathrm{keV}$. In general agreement with the CNS calculations, the lowest calculated $27^{-}$state is dominated by the $\pi\left(g_{7 / 2}^{5} d_{5 / 2}^{4} h_{11 / 2}^{1}\right) \otimes$ $v h_{11 / 2}^{-2}$ configuration (amplitude 59\%), while the $26_{1,2}^{+}$states are dominated by the $\pi\left(g_{7 / 2}^{5} d_{5 / 2}^{3} h_{11 / 2}^{2}\right) \otimes v h_{11 / 2}^{-2}$ configuration, implying thus a $\pi\left(d_{5 / 2}^{3} h_{11 / 2}^{2}\right) \rightarrow \pi\left(d_{5 / 2}^{4} h_{11 / 2}^{1}\right)$ transition in the $26_{1,2}^{+} \rightarrow 27^{-}$decay. One can then estimate the relative quasiparticle excitation energy between the $h_{11 / 2}$ and $d_{5 / 2}$ proton orbitals as $E\left(\pi h_{11 / 2}-\pi d_{5 / 2}\right) \approx 370 \mathrm{keV}$, which is not far from the $\approx 300-\mathrm{keV}$ difference between the $h_{11 / 2}$ and $g_{7 / 2}$ proton orbitals extracted from the decay of the $20^{+}$isomer. These values can be compared with the difference between the effective single-particle energies of around $1.5 \mathrm{MeV}$ plotted in Fig. 7(b), showing important mixing in these two states. We have also calculated the yrast $32^{+}$state, which is dominated by the $\pi\left(g_{7 / 2}^{8} h_{11 / 2}^{2}\right) \otimes v h_{11 / 2}^{-2}$ configuration (amplitude 78\%), 
which appear to be consistent with the CNS calculations. In addition, it is interesting to verify if the variation of the neutron and proton effective single-particle energies with the filling the neutron $h_{11 / 2}$ orbit is in agreement with the tensor mechanism proposed in Ref. [26]. As one can see in Fig. 7, the SM Hamiltonian shows that as more neutrons occupy the $h_{11 / 2}$ orbit, the $g_{7 / 2}\left(j=4-\frac{1}{2}\right)$ and $h_{11 / 2}\left(j=5+\frac{1}{2}\right)$ orbitals move apart in both the $v v$ [Fig. 7(a)] and $v \pi$ channels [Fig. 7(b)], as expected.

\section{SUMMARY}

In summary, the present paper reports the discovery of several new spherical states in ${ }^{140} \mathrm{Nd}$, supporting the existence of coexisting spherical and triaxial states up to high spins in the $A=140$ mass region. In the CNS calculations, the lowest $20^{+}, 27^{-}$, and $32^{+}$states are formed as maximally aligned in the configurations with two $h_{11 / 2}$ neutron holes and 0,1 , and 2 protons in $h_{11 / 2}$. This is consistent with the shell model for the $27^{-}$and $32^{+}$states, while the lowest $20^{+}$state appears to be much more mixed in the shell model. Furthermore, the comparison between the experimental level energies and those calculated in the shell model allowed us to establish the relative energy between several proton and neutron orbitals at high energy and spins. A good agreement between experimental data and shell-model calculations for the spherical states is obtained up to high spins, which gives credit to the newly developed effective interaction for the $50 \leqslant N, Z \leqslant 82$ mass region. However, while the comparison with the shell-model results suggests that the observed $27^{(-)}$ state at $11204 \mathrm{keV}$ corresponds to the fully aligned state of the $\pi\left(h_{11 / 2}\right)^{1} \otimes v\left(h_{11 / 2}\right)^{-2}$ configuration, the CNS calculations rather suggest that this is the configuration of band $D 3$, for which the highest spin states are not observed at present. Further experiments giving more details of the level scheme would be necessary to resolve this issue.

\section{ACKNOWLEDGMENTS}

This work has been supported by the EU-FP7-IA project ENSAR (No. 262010) and the Academy of Finland (CoE in Nuclear and Accelerator-Based Physics). The GAMMAPOOL European Spectroscopy Resource is thanked for the loan of detectors for JUROGAM II. The authors acknowledge the support from the Helmholtz Association through the Nuclear Astrophysics Virtual Institute NAVI (No. VH-VI-417) (H.N.) and the Swedish Research Council (I.R.).
[1] P. Möller, J. R. Nix, W. D. Myers, and W. J. Swiatecki, At. Data Nucl. Data Tables 59, 185 (1995).

[2] A. Neußer et al., Phys. Rev. C 70, 064315 (2004).

[3] C. M. Petrache et al., Phys. Rev. C 61, 011305(R) (1999).

[4] C. M. Petrache et al., Phys. Rev. C 86, 044321 (2012).

[5] C. M. Petrache et al., Phys. Rev. C 72, 064318 (2005).

[6] R. Leguillon et al., Phys. Rev. C 88, 014323 (2013).

[7] T. Zerrouki et al., Eur. Phys. J. A 51, 50 (2015).

[8] C. M. Petrache, I. Ragnarsson, H.-L. Ma, R. Leguillon, T. Zerrouki, D. Bazzacco, and S. Lunardi, Phys. Rev. C 91, 024302 (2015).

[9] C. M. Petrache et al., Phys. Rev. C 74, 034304 (2006).

[10] M. Ferraton et al., Eur. Phys. J. A 35, 167 (2008).

[11] A. Vancraeyenest et al., Phys. Rev. C 87, 064303 (2013).

[12] R. D. Page et al., Nucl. Instrum. Methods B 204, 634 (2003).

[13] M. Leino et al., Nucl. Instrum. Methods B 99, 653 (1995).

[14] P. J. Nolan, F. A. Beck, and D. B. Fossan, Annu. Rev. Nucl. Part. Sci. 44, 561 (1994).
[15] R. Léguillon, Ph.D. thesis, Paris Sud University, Orsay, 2013, http://tel.archives-ouvertes.fr/tel-00932123.

[16] A. V. Afanasjev, D. B. Fossan, G. J. Lane, and I. Ragnarsson, Phys. Rep. 322, 1 (1999).

[17] T. Bengtsson and I. Ragnarsson, Nucl. Phys. A 436, 14 (1985).

[18] A. V. Afanasjev and I. Ragnarsson, Nucl. Phys. A 591, 387 (1995).

[19] B. G. Carlsson and I. Ragnarsson, Phys. Rev. C 74, 011302(R) (2006).

[20] A. Bohr, I. Hamamoto, and B. R. Mottelson, Phys. Scr. 26, 267 (1982).

[21] T. Bengtsson, Nucl. Phys. A 512, 124 (1990).

[22] A. Gniady, A. Caurier, and F. Nowacki (unpublished).

[23] K. Sieja, G. Martinez-Pinedo, L. Coquard, and N. Pietralla, Phys. Rev. C 80, 054311 (2009).

[24] E. Caurier, F. Nowacki, A. Poves, and K. Sieja, Phys. Rev. C 82, 064304 (2010).

[25] F. Nowacki and A. Caurier (unpublished).

[26] T. Otsuka, T. Suzuki, R. Fujimoto, H. Grawe, and Y. Akaishi, Phys. Rev. Lett. 95, 232502 (2005) 